\title{
First-line treatment of metastatic melanoma: role of nivolumab
}

Jeremy Force'

April KS Salama ${ }^{1,2}$

'Division of Hematology/Oncology, Duke University Medical Center, Durham, NC, USA; ${ }^{2}$ Division of Medical Oncology, Duke University Medical Center, Durham, NC, USA
This article was published in the following Dove Press journal:

ImmunoTargets and Therapy

13 February 2017

Number of times this article has been viewed
Correspondence: April KS Salama Division of Medical Oncology, Department of Medicine, Duke University, DUMC 3198, Durham, NC 277I0, USA

$\mathrm{Tel}+19196819509$

Fax + I 9196819599

Email april.salama@duke.edu
Abstract: Historically, the median overall survival of metastatic melanoma patients was less than 1 year and long-term survivors were rare. Recent advances in therapies have dramatically shifted this landscape with increased survival rates and the real possibility that long-term disease control is achievable. Advances in immune modulators, including cytotoxic T-lymphocyte antigen- 4 and programmed death- 1 based treatments, have been an integral part of this success. In this article, we review previous and recent therapeutic developments for metastatic melanoma patients. We discuss advances in immunotherapy while focusing on the use of nivolumab alone and in combination with other agents, including ipilimumab in advanced melanoma. One major goal in melanoma research is to optimize combination strategies allowing for more patients to experience benefit while minimizing toxicity. A better understanding of the optimal sequencing, combinations, and mechanisms underlying the development of resistance may provide evidence for rational clinical trial designs of novel immunotherapy strategies in melanoma and other cancer subtypes.

Keywords: PD-1, immunotherapy, pembrolizumab, PD-L1, resistance, checkpoint, BRAF

\section{Introduction}

Melanoma is a neoplasm of melanocytes that usually occurs in the skin, but rarely may also begin in the uvea or other sites. Melanoma of the skin is the sixth most common cancer in the US, and it is estimated that there will be 73,870 new cases of melanoma and 9,940 melanoma-related deaths in $2015 .{ }^{1}$ Once melanoma metastasized to distant sites, the historic 5-year survival rates were low and median overall survival (OS) was previously less than 1 year. $^{2}$ Before 2011 , there were limited effective systemic therapies available. Cytotoxic agents, biochemotherapy, and high-dose interleukin-2 (HDIL-2) were among the backbone of treatment options, but none had demonstrated an OS benefit in a randomized study. ${ }^{3}$

Dacarbazine, one of the first drugs approved by the US Food and Drug Administration (FDA) for the treatment of metastatic melanoma, demonstrated an overall response rate of $\sim 20 \%$ with no impact on survival. ${ }^{4} \mathrm{HDIL}-2$, the only other agent approved for metastatic disease prior to 2011, has shown low objective response rates, albeit with the potential for durable responses in a small subset of patients. However, the significant toxicities associated with HDIL-2 limit its use in patients with extensive comorbidities, and administration is usually restricted to specialized centers. ${ }^{5}$

More recent advances in molecularly targeted therapies have shown great promise in the treatment of advanced melanoma. $B R A F$ mutations are present in up to $57 \%$ 
of metastatic melanomas, with the most common activating mutations being V600E and V600K. ${ }^{6}$ Metastatic melanoma patients whose tumors harbor a $B R A F$ mutation have been shown to respond to the selective BRAF inhibitors dabrafenib and vemurafenib. ${ }^{7,8}$ More recently, combination therapy with BRAF and MEK inhibitors has resulted in improved response rates and in some studies OS when compared to BRAF inhibitor monotherapy. ${ }^{9-11}$ Dual inhibition now represents a standard of care option for patients with $B R A F$-mutated melanoma. Challenges remain, however, as the majority of melanomas ultimately develop resistance and additional therapeutic options are needed.

An increased understanding of immunoregulatory mechanisms has also led to the development of a novel class of immune therapies, collectively termed immune checkpoint inhibitors. One of the first agents in this class, the anti-cytotoxic T-lymphocyte antigen-4 (CTLA-4) antibody ipilimumab received regulatory approval for the treatment of advanced melanoma in 2011, and has the potential for durable responses in $\sim 20 \%$ of patients. ${ }^{12,13}$ The subsequent development of antibodies blocking the programmed death-1 (PD-1) pathway, including the FDA-approved agents pembrolizumab and nivolumab, has also seen clinical success within the field of melanoma, with higher response rates and less toxicity when compared to ipilimumab alone. ${ }^{14,15} \mathrm{~A}$ more recent development using dual checkpoint blockade with concurrent ipilimumab and nivolumab has further increased the potential for clinical benefit, though concerns regarding higher rates of toxicity remain. ${ }^{16}$ Together, these developments represent a mainstay of systemic therapy for patients with advanced melanoma.

Additional advances include the development of talimogene laherparepvec, a herpes simplex virus type 1-derived oncolytic immunotherapy that was recently approved for the treatment of unresectable cutaneous, subcutaneous, and nodal lesions in patients with postoperative recurrent melanoma based on efficacy data from a Phase III trial. ${ }^{17}$ Talimogene laherparepvec is designed to selectively replicate within tumors and produce granulocyte macrophage colony-stimulating factor to theoretically enhance systemic antitumor immune responses.

Finally, innovations in additional immunotherapeutic strategies including adoptive cell transfer of tumor-infiltrating lymphocytes, or targeting other novel immunologic checkpoints, such as programmed death ligand 1 (PD-L1) and LAG-3 or the co-stimulatory molecules OX40 and 4-1BB, are at various phases of clinical development and have the potential to benefit more patients in the future. ${ }^{18}$ With adoptive cell transfer, $T$ cells are isolated from a resected melanoma, expanded ex vivo, and infused back into the same melanoma patient. ${ }^{19}$ This therapeutic strategy was one of the first therapies to show that human $T$ cells could mediate the rejection of metastatic melanoma, and subsequent studies have shown the potential for durable responses. ${ }^{20,21}$ Recent work is focused on using traditional lymphodepleting preparative regimens in conjunction with immune checkpoint inhibition in hopes of further augmenting antitumor activity (NCT02621021).

Anti-PD-L1 monoclonal antibodies, including atezolizumab, avelumab, and durvalumab, are being studied either alone or in combination in several tumor types, including melanoma. In an initial dose-escalation study of the PD-L1 antibody BMS-936559, durable responses were seen in a cohort of 55 melanoma patients, and side effects were manageable. ${ }^{22}$ More recent studies have explored the feasibility of combination strategies, including a report of atezolizumab combined with vemurafenib, which demonstrated early promise in a Phase I study. ${ }^{23}$

\section{PD-I-based therapy in melanoma: preclinical rationale}

Major advancements have occurred in our understanding of checkpoint blockade involving CTLA-4 and PD-1 (Figure 1). These discoveries have allowed for therapeutic developments of novel checkpoint inhibitors including ipilimumab, pembrolizumab, and nivolumab, among others.

PD-1 is a type I transmembrane glycoprotein composed of an immunoglobulin (Ig) V-type extracellular domain. ${ }^{24}$ The human and murine $\mathrm{PD}-1$ receptors have $60 \%$ protein homology and $70 \%$ homology at the nucleotide coding region. ${ }^{24}$ PD-1 lacks a cysteine residue required for homodimerization to other members of the $\mathrm{B} 7$ family. ${ }^{25} \mathrm{PD}-1$ is primarily located on the surface of activated T cells, B cells, NK cells, and macrophages. PD-1 contains two cytoplasmic tyrosine residues that constitute an immunoreceptor tyrosine-based inhibitory motif and an immunoreceptor tyrosine-based switch motif. ${ }^{24}$ PD-1 is activated upon interaction with one of its ligands. The PD receptor/ligand complex will inhibit T-cell signaling by immunoreceptor tyrosine-based switch motif-mediated recruitment of the protein tyrosine phosphatases SHP-1 and SHP-2, which dephosphorylate downstream T-cell receptor effector molecules leading to T-cell apoptosis. ${ }^{26}$

The immune checkpoints CTLA-4 and PD-1 were discovered and classified within the B7 family in $1992 .{ }^{24}$ Seven years later, it was determined that PD-1 binds to two ligands, PD-L1 and PD-L2. ${ }^{27}$ The PD receptor/ligand complex has been studied extensively in autoimmunity, tumor immunity, allergy, and 

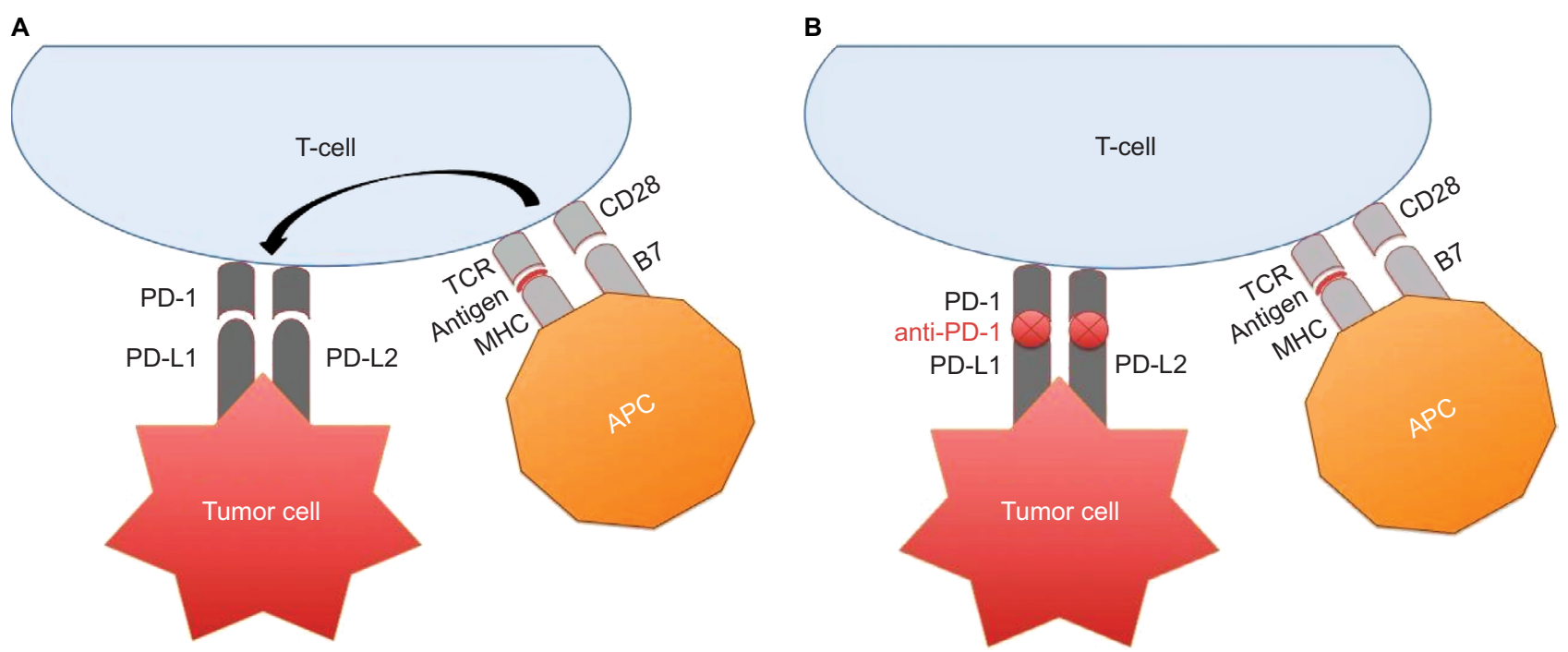

Figure I (A) APC presents tumor-associated antigens to circulating T cells via MHC complex binding to TCR. However, a co-stimulatory signal, provided upon the binding of CD28/B7 is required for T cell activation. Following T-cell stimulation, PD-I expression occurs. PD-LI and PD-L2 expressed on tumor cells can interact with PD-I on $T$ cells in the tumor microenvironment causing T-cell down regulation and tumor immune evasion. (B) Anti-PD-I inhibits PD-I binding to PD-LI and PD-L2 allowing the immune system to recognize tumors as foreign and induce immune-mediated tumor cellular death.

Abbreviations: APC, antigen-presenting cell; MHC, major histocompatibility complex; TCR, T-cell receptor; CD, cluster designation; PD-I, programmed death-I; PD-L, programmed cell death ligand.

organ transplantation. ${ }^{28-31}$ Under normal conditions, $\mathrm{PD}-1$ plays an important role in preventing excessive immune reactions by negatively regulating the functions of autoreactive $\mathrm{T}$ cells.

Based on these discoveries, it was hypothesized that interruption of the $\mathrm{PD}$ receptor/ligand complex would unleash the immune system to destroy cancer cells. Anti-PD-1 monoclonal antibodies were first studied in murine cancer models in $2005 .{ }^{32}$ Compared to wild-type mice, intrasplenic injection of poorly immunogenic B16 melanoma cells into PD-1 deficient mice, followed by PD-L1 induction on tumor cells, showed enhanced induction of splenic effector T cells, increased T-cell proliferation and cytokine production, and augmented homing of effector T cells to tumor sites. Similar experiments were carried out with CT26 colon cancer cells that demonstrated that there was decreased hematogenous dissemination of colon cancer to the lungs. ${ }^{32}$ These experiments helped lay the groundwork that anti-PD-1 blocks tumor growth by inhibiting PD-1-induced immunosuppression, thus allowing $\mathrm{T}$ cells to proliferate and attack cancer cells.

\section{Nivolumab pharmacology, mechanism of action, and pharmacokinetics}

Nivolumab was developed under several names including MDX-1106, BMS-936558, and ONO-4538. Nivolumab is a fully human IgG4 monoclonal antibody against programmed death receptor-1. It contains an engineered hinge region mutation that prevents exchange of IgG4 molecules. ${ }^{33}$ The IgG4 isotype minimizes antibody-dependent and complementmediated cellular cytotoxicity. As monotherapy, nivolumab is administered at a flat dose of $240 \mathrm{mg}$ every 2 weeks.

Nivolumab pharmacokinetics were studied in 35 patients by determining anti-PD-1 serum concentrations from enzyme-linked immunosorbent assays. After initiation of nivolumab infusion, the median time to peak concentration of nivolumab was 1-4 hours. Pharmacokinetics of nivolumab was linear. A dose-proportional increase in the peak concentration and area under the curve calculated from days 1-14 were noted to be in the dose range of $0.1-10.0 \mathrm{mg} / \mathrm{kg}$. The mean elimination half-life is 26.7 days, and steady-state concentrations of nivolumab were reached by 12 weeks when administered at $3 \mathrm{mg} / \mathrm{kg}$ every 2 weeks. ${ }^{34,35}$

Pharmacodynamic analysis of nivolumab was evaluated on peripheral blood mononuclear cells that were isolated from patients at baseline and after the first treatment cycle. Estimated occupancy of nivolumab on PD-1 receptor on circulating CD3 + T cells was assessed by flow cytometry from 65 patients with melanoma who were treated with one cycle of nivolumab at a dose of $0.1-10.0 \mathrm{mg} / \mathrm{kg}$ intravenously every 2 weeks. Nivolumab's affinity for PD-1 receptor was reported to occupy $\sim 70 \%$ of circulating CD3+T-cell PD-1 molecules. ${ }^{34,35}$

\section{Nivolumab response and efficacy: clinical data}

Ipilimumab was the first checkpoint inhibitor to gain FDA approval by demonstrating a survival benefit in metastatic 
melanoma patients in a prospectively randomized Phase III clinical trial. ${ }^{36}$ Pembrolizumab was initially approved for ipilimumab-refractory metastatic melanoma patients; however, the indication was expanded to the first-line setting based on KEYNOTE-006 results. ${ }^{14,37}$ Similarly, nivolumab was FDA approved for advanced melanoma patients refractory to other therapies, and then subsequently received first-line approval in 2015..$^{15,38,39}$ The pivotal CheckMate 067 clinical trial demonstrated a significant progression-free survival (PFS) benefit when nivolumab was combined with ipilimumab. Dual checkpoint blockade with ipilimumab and nivolumab was FDA approved for the first-line treatment of $B R A F$ wild-type advanced melanoma and later expanded to include patients with $B R A F$-mutated disease in January 2016. ${ }^{16,40}$

Nivolumab has been extensively studied in several advanced/metastatic carcinoma settings. Of note, many of these clinical trials evaluating the safety and efficacy of nivolumab in advanced and metastatic melanoma patients included patients with $B R A F$ mutations. ${ }^{41}$

\section{Nivolumab efficacy in chemotherapy refractory ipilimumab-naïve advanced melanoma}

The chemotherapy-refractory ipilimumab-naïve melanoma cohort from the study by Topalian et al was expanded to investigate OS, response duration, and long-term safety in patients who had received at least 14 months of nivolumab. ${ }^{35,38}$ The median OS was 16.8 months and the 2-year OS rate was $43 \%$ (Table 1). Among those with an objective response (31\%), the estimated median response duration was 2 years. This initial study reported grade 3-4 adverse events (AEs) of $\geq 5 \%$ in the nivolumab 1 and $3 \mathrm{mg} / \mathrm{kg}$ cohorts as being fatigue (5\%) and dyspnea (5\%). ${ }^{35}$ However, long-term follow up revealed that $22 \%$ of patients experienced grade $3-4$ AEs including fatigue, diarrhea, lymphopenia, and abdominal pain. Nivolumabassociated immune-related AEs that occurred in $\leq 3 \%$ of the total melanoma population included pneumonitis, colitis, renal failure, hepatitis, hypophysitis, thyroiditis, uveitis, and tubulointerstitial nephritis. The exposure-adjusted toxicity rates were not cumulative. ${ }^{38}$

\section{Nivolumab efficacy in the first-line metastatic setting}

CheckMate 066 was an international, multicenter, doubleblind, randomized, controlled, Phase III study that enrolled 418 untreated $B R A F$ wild-type unresectable stage III or IV melanoma patients to receive either nivolumab $3 \mathrm{mg} / \mathrm{kg}$ every 2 weeks with dacarbazine placebo every 3 weeks or nivolumab placebo every 2 weeks with dacarbazine 1,000 $\mathrm{mg} / \mathrm{m}^{2}$ every 3 weeks until disease progression or unacceptable toxicity. ${ }^{42}$ The primary endpoint was OS. The median OS was not reached in the nivolumab group, but it was 10.8 months in those patients who received dacarbazine. The OS rate at 1 year in the nivolumab- and dacarbazine-treated groups were $73 \%$ and $42 \%$, respectively (Table 1 ). There were $7.6 \%$ complete responses (CR) with nivolumab and $1 \%$ in dacarbazine-treated patients. Among those with an objective response, the median duration of response was not reached in the nivolumab group, but it was 6 months in those treated with dacarbazine. Forty-seven percent of patients treated with nivolumab had progressive disease compared to $85 \%$ in the dacarbazine cohort. Although the rates of treatment-related AEs were similar with nivolumab (74.3\%) and dacarbazine (75.6\%), the incidence of grade 3-4 AEs were different at $11.7 \%$ and $17.6 \%$, respectively. Additionally, the percentage

Table I Nivolumab monotherapy and combination with ipilimumab efficacy

\begin{tabular}{|c|c|c|c|c|c|c|c|}
\hline Monotherapy & Patients, n & ORR & CR & $\begin{array}{l}\text { Median } \\
\text { PFS ( } 3 \mathrm{mg} / \mathrm{kg})\end{array}$ & Median OS & I-Year OS & 2-Year OS \\
\hline Topalian et $\mathrm{al}^{38}$ & 107 & $31 \%$ & NR & 9.7 months & 16.8 months & $62 \%$ & $43 \%$ \\
\hline $\begin{array}{l}\text { Robert et al }{ }^{42} \\
\text { (previously untreated) }\end{array}$ & 418 & $40 \%$ & $7.6 \%$ & 5.I months & Not reached & $73 \%$ & NR \\
\hline $\begin{array}{l}\text { Weber et al }{ }^{39} \\
\text { (ipilimumab refractory) }\end{array}$ & 405 & $31.7 \%$ & $3.3 \%$ & 4.7 months & NR & NR & NR \\
\hline Combination therapy & Patients, n* & ORR & CR & Median PFS & Median OS & I-Year OS & 2-Year OS \\
\hline $\begin{array}{l}\text { Wolchok et al }{ }^{43} \\
\text { (all dose levels) }\end{array}$ & 53 & $40 \%$ & $9.4 \%$ & NR & NR & NR & NR \\
\hline & & & & $\begin{array}{l}\text { BRAF WT } \\
\text { not reached }\end{array}$ & & & \\
\hline Postow et $\mathrm{al}^{40,44}$ & 95 & $61 \%$ & $22 \%$ & $\begin{array}{l}\text { BRAF mut } \\
8.5 \text { months }\end{array}$ & Not reached & $73 \%$ & $64 \%$ \\
\hline Larkin et $\mathrm{al}^{4 \mathrm{I}}$ & 314 & $57.6 \%$ & $11.5 \%$ & Il.5 months & Not reached & NR & NR \\
\hline
\end{tabular}

Note: *Receiving concurrent ipilimumab + nivolumab.

Abbreviations: ORR, objective response rate; CR, complete response; PFS, progression-free survival; OS, overall survival; NR, not reported; WT, wild type. 
of patients who discontinued nivolumab were $6.8 \%$ compared to $11.7 \%$ with dacarbazine. ${ }^{42}$

\section{Nivolumab efficacy in ipilimumab- refractory advanced melanoma}

CheckMate 037 was an international, multicenter, openlabel, Phase III trial that enrolled 631 unresectable stage IIIC or metastatic melanoma patients who had progressed on ipilimumab. Patients were randomized to receive either nivolumab $3 \mathrm{mg} / \mathrm{kg}$ every 2 weeks or investigator's choice chemotherapy (ICC) (dacarbazine $1000 \mathrm{mg} / \mathrm{m}^{2}$ every 3 weeks or combination carboplatin AUC 6 with paclitaxel $175 \mathrm{mg} / \mathrm{m}^{2}$ every 3 weeks) until disease progression or unacceptable toxicity. ${ }^{39}$ Sixty-four percent of the patients had derived no benefit from ipilimumab monotherapy. At the first interim analysis, patients had received nivolumab or ICC for a median of 5.3 and 2.0 months, respectively. The number of patients discontinuing treatment in the nivolumab arm was $52 \%$ versus $82 \%$ in the ICC group, with the majority of patients discontinuing therapy due to disease progression.

Objective responses were observed in $31.7 \%$ of the patients in the nivolumab cohort compared to $10.6 \%$ of the patients in the ICC group (Table 1). Best overall response rates (CR and $\mathrm{PR}$ ) in the nivolumab group were 3\% and $28 \%$ versus $0 \%$ and $11 \%$ for the ICC cohort, respectively. Time to response in those who received nivolumab was 2.1 months compared to 3.5 months with ICC. Median duration of response had not been reached in the nivolumab group compared to 3.5 months in patients who received ICC. Eight percent of the patients in the nivolumab group experienced grade $3 \mathrm{AEs}$ and $1 \%$ had a grade $4 \mathrm{AE} .^{39}$

\section{Efficacy of dual checkpoint blockade with nivolumab and ipilimumab}

Early phase studies reporting the results of dual checkpoint blockade demonstrated considerable promise. ${ }^{43}$ As a result, a randomized Phase II study was conducted that enrolled 142 untreated metastatic melanoma patients to receive combination ipilimumab $3 \mathrm{mg} / \mathrm{kg}$ with nivolumab $1 \mathrm{mg} / \mathrm{kg}$ or placebo every 3 weeks for four doses followed by nivolumab $3 \mathrm{mg} / \mathrm{kg}$ or placebo every 2 weeks until disease progression or unacceptable toxicity. ${ }^{40}$ The primary endpoint was defined as the rate of investigator-assessed objective response. Approximately $58 \%$ of patients received at least four doses of combination therapy and
$70 \%$ received all four doses of ipilimumab monotherapy. The investigator-assessed objective response rate was $61 \%$ in the combination group compared to $11 \%$ in the ipilimumab monotherapy cohort (Table 1). Notably, 16 patients $(22 \%)$ achieved a CR in the combination group, and no patients obtained a CR with ipilimumab alone. Recently, survival analysis was updated in this trial reporting 1 - and 2-year OS of $73 \%$ and $64 \%$, respectively. ${ }^{44}$ Rates of all investigator-assessed treatment-related AEs in the combination cohort and the ipilimumab-alone group were similar at $91 \%$ and $93 \%$, respectively. However, the combination group reported higher rates of grade 3-4 AEs at $54 \%$ compared to $24 \%$ in the ipilimumab monotherapy group. Interestingly, the majority of AEs occurred while receiving combination treatment rather than being associated with nivolumab monotherapy maintenance. The most common grade 3-4 AEs in those treated with ipilimumab and nivolumab were colitis (17\%), diarrhea (11\%), and transaminitis (11\%). The immune-related AEs were skin, gastrointestinal, endocrine, and hepatic in origin and were observed more frequently in the combination cohort compared to the ipilimumab-alone group. ${ }^{40}$

CheckMate 067 was a subsequent Phase III study that enrolled 945 untreated unresectable stage III or metastatic melanoma patients to one of three arms: 1) nivolumab $1 \mathrm{mg} / \mathrm{kg}$ with ipilimumab $3 \mathrm{mg} / \mathrm{kg}$ every 3 weeks for four cycles followed by nivolumab $3 \mathrm{mg} / \mathrm{kg}$ every 2 weeks (combination treatment with nivolumab maintenance); 2) nivolumab $3 \mathrm{mg} / \mathrm{kg}$ every 2 weeks with ipilimumab placebo every 3 weeks (nivolumab monotherapy); or 3) ipilimumab $3 \mathrm{mg} / \mathrm{kg}$ every 3 weeks for four cycles with nivolumab placebo every 2 weeks (ipilimumab monotherapy) until disease progression or unacceptable toxicity. ${ }^{16}$ The primary endpoints were PFS and OS. The median PFS and investigator-assessed objective response rates in the combination treatment with nivolumab maintenance, nivolumab monotherapy, and ipilimumab monotherapy cohorts were $11.5,6.9$, and 2.9 months and $57.6 \%, 43.7 \%$, and $19 \%$, respectively (Table 1). All treatment-related AEs were seen in $95.5 \%$ of patients treated with the combination. The rates of AEs were somewhat lower in the monotherapy cohorts at $82.1 \%$ with nivolumab and $86.2 \%$ with ipilimumab. AEs that led to treatment discontinuation were seen in $36.4 \%$ of patients in the combination group, $7.7 \%$ of patients in the nivolumab arm, and $14.8 \%$ of patients in the ipilimumab arm (Table 2). Adverse event profiles were similar to previously reported studies, and importantly no combination treatmentrelated deaths were reported. ${ }^{16}$ 
Table 2 Nivolumab monotherapy and combination with ipilimumab side effect profile

\begin{tabular}{|c|c|c|c|c|c|c|}
\hline \multirow[t]{2}{*}{ Adverse event category } & \multicolumn{2}{|l|}{ Nivolumab } & \multicolumn{2}{|l|}{ Ipilimumab } & \multicolumn{2}{|c|}{ Nivolumab + ipilimumab } \\
\hline & Any, n (\%) & Grades 3-4, n (\%) & Any, n (\%) & Grades 3-4, n (\%) & Any, n (\%) & Grades 3-4, n (\%) \\
\hline \multicolumn{7}{|l|}{ Endocrine } \\
\hline Hypothyroidism & $27(8.6)$ & 0 & $13(4.2)$ & $\mathrm{I}(0.3)$ & $47(15)$ & $\mathrm{I}(0.3)$ \\
\hline Hyperthyroidism & $13(4.2)$ & 0 & $3(1)$ & 0 & $31(9.9)$ & $3(1)$ \\
\hline Hypophysitis & $2(0.6)$ & $\mathrm{I}(0.3)$ & $12(3.9)$ & $6(1.9)$ & $24(7.7)$ & $5(1.6)$ \\
\hline Pyrexia & $18(5.8)$ & 0 & $21(6.8)$ & $\mathrm{I}(0.3)$ & $58(6.8)$ & $\mathrm{I}(0.3)$ \\
\hline \multicolumn{7}{|l|}{ Gastrointestinal } \\
\hline Elevated ALT/AST & $24(7.7)$ & $7(2.2)$ & $23(7.3)$ & $7(2.2)$ & $103(33)$ & $45(14.4)$ \\
\hline Diarrhea & $60(19.2)$ & $7(2.2)$ & $103(33.1)$ & $19(6.1)$ & I $38(44.1)$ & $29(9.3)$ \\
\hline Colitis & $4(1.3)$ & $2(0.6)$ & $36(11.6)$ & $27(8.7)$ & $37(I I .8)$ & $24(7.7)$ \\
\hline Nausea & $4 \mid(\mid 3.1)$ & 0 & $50(16.1)$ & $2(0.6)$ & $81(25.9)$ & $7(2.2)$ \\
\hline Vomiting & $20(6.4)$ & $\mathrm{I}(0.3)$ & $23(7.4)$ & $\mathrm{I}(0.3)$ & $48(15.3)$ & $8(2.6)$ \\
\hline Decreased appetite & $34(10.9)$ & 0 & $39(12.5)$ & I (0.3) & $56(17.9)$ & $4(1.3)$ \\
\hline \multicolumn{7}{|l|}{ Musculoskeletal } \\
\hline Arthralgia & $24(7.7)$ & 0 & $19(6.1)$ & 0 & $33(10.5)$ & $\mathrm{I}(0.3)$ \\
\hline \multicolumn{7}{|l|}{ Neuropsychiatric } \\
\hline Fatigue & $107(34.2)$ & $4(1.3)$ & $87(28)$ & $3(1)$ & $110(35.1)$ & $13(4.2)$ \\
\hline Headache & $23(7.3)$ & 0 & $24(7.7)$ & I (0.3) & $32(10.2)$ & I (0.3) \\
\hline \multicolumn{7}{|l|}{ Pulmonary } \\
\hline Pneumonitis & $4(1.3)$ & I (0.3) & $5(1.6)$ & I (0.3) & $20(6.4)$ & $3(1)$ \\
\hline Dyspnea & $14(4.5)$ & $\mathrm{I}(0.3)$ & $13(4.2)$ & 0 & $32(10.2)$ & $2(0.6)$ \\
\hline \multicolumn{7}{|l|}{ Skin } \\
\hline Rash & 81 (25.9) & $2(0.6)$ & $102(32.8)$ & $6(1.9)$ & $126(40.3)$ & $15(4.8)$ \\
\hline Vitiligo & $23(7.3)$ & $\mathrm{I}(0.3)$ & $12(3.9)$ & 0 & $21(6.7)$ & 0 \\
\hline Pruritus & $59(18.8)$ & 0 & $110(35.4)$ & I (0.3) & $104(33.2)$ & $6(1.9)$ \\
\hline $\begin{array}{l}\text { Discontinuation due to } \\
\text { treatment-related } \mathrm{AE}\end{array}$ & $24(7.7)$ & $16(5.1)$ & $46(14.8)$ & $4 \mid(\mid 3.2)$ & $114(36.4)$ & $92(29.4)$ \\
\hline
\end{tabular}

Note: Data from Robert C, Long GV, Brady B, et al. Nivolumab in previously untreated melanoma without BRAF mutation. N Engl J Med. 20I5;372(4):320-330.42

Abbreviations: $A E$, adverse event; $A L T$, alanine aminotransferase; $A S T$, aspartate aminotransferase.

\section{Nivolumab safety and tolerability Nivolumab monotherapy}

Initial Phase I trials investigated the use of nivolumab across multiple tumor types. ${ }^{33}$ Two large multicenter Phase I trials enrolled 503 advanced cancer patients to receive dose escalations of nivolumab. ${ }^{22,35}$ Both the trials reported that nivolumab was well tolerated. Topalian et al showed that common treatment-related AEs occurring in $\geq 5 \%$ of patients included fatigue (16\%), infusion reaction (10\%), diarrhea $(9 \%)$, arthralgia (7\%), rash (7\%), pruritus $(6 \%)$, and nausea $(6 \%) .{ }^{35}$ The majority of AEs occurred in the patients who received 1,3 , or $10 \mathrm{mg} / \mathrm{kg}$ of nivolumab with most infusion reactions observed at the $10 \mathrm{mg} / \mathrm{kg}$ dose. A dose effect on AE profile was observed with higher doses of nivolumab causing increased AEs. Serious AEs of any grade were seen in eleven patients (5\%) and only developed in those who received $\geq 1 \mathrm{mg} / \mathrm{kg}$ nivolumab. Nineteen patients ( $9 \%$ ) had grade $\geq 3$ serious AEs, all of which occurred at a rate of $1 \%$. These included vomiting, fatigue, hyperglycemia, infusion reaction, adrenal insufficiency, and endophthalmitis. There was one (1\%) drug-related death.
Nivolumab safety was also tested in chemotherapy- and ipilimumab-refractory advanced melanoma patients. ${ }^{45}$ The safety, tolerability, and toxicity profiles in the initial Phase I trials were similar to subsequent Phase III trials (Table 2). ${ }^{16,22,35}$

\section{Safety of dual checkpoint blockade with nivolumab and ipilimumab}

The investigation of the combination of nivolumab and ipilimumab was initially described in an innovative Phase I clinical trial design where 86 melanoma patients received concurrent therapy or sequenced treatment. ${ }^{43}$ Fifty-three patients received concurrent therapy and 33 received sequenced treatment. All the patients were followed up for 2.5 years after the initiation of therapy. ${ }^{43}$

Overall, $93 \%$ of patients developed a treatment-related AE. Grade 3-4 treatment-related AEs in the concurrent and sequenced cohorts were $53 \%$ and $18 \%$ of patients, respectively. Grade $3-4$ events occurring in $\geq 5 \%$ of patients from cohort 2 (nivolumab $1 \mathrm{mg} / \mathrm{kg}$ and ipilimumab $3 \mathrm{mg} / \mathrm{kg}$ ) and cohort $2 \mathrm{a}$ (nivolumab $3 \mathrm{mg} / \mathrm{kg}$ and ipilimumab $1 \mathrm{mg} / \mathrm{kg}$ ) were 
rash, diarrhea, vomiting, colitis, elevated amylase and lipase, transaminitis, elevated serum creatinine, pneumonitis, and uveitis. Six of 28 patients (21\%) had dose-limiting grade 3-4 AEs with concurrent therapy. Serious treatment-related AEs in the concurrent and sequenced-based regimens were $49 \%$ and $21 \%$, respectively. Eleven patients (21\%) discontinued treatment in the concurrent therapy cohort compared to three patients $(9 \%)$ receiving sequenced treatment. A maximum tolerated dose was selected from cohort 2 using nivolumab $1 \mathrm{mg} / \mathrm{kg}$ and ipilimumab $3 \mathrm{mg} / \mathrm{kg} .{ }^{43}$ Subsequent results of randomized Phase II and III studies with the combination demonstrated a similar AE profile (Table 2). ${ }^{16,40}$

\section{Optimizing nivolumab use alone and in combination}

The use of PD-1-based therapy is now a mainstay of therapy for advanced melanoma, with both nivolumab and pembrolizumab having regulatory approval. Additionally, the combination of ipilimumab with nivolumab was recently approved, although marked differences in the toxicity profile of the combination require careful consideration and patient selection.

Currently, there are no definitive predictive markers of nivolumab response in any cancer subtype used in standard of care practice. Commercially available antibodies against PD-L1 lack specificity, and controversy exists as to whether IHC or mRNA PD-L1 expression is the most optimal way to capture PD-L1-expressing tumors. ${ }^{46,47}$ Data from Phase II/III studies suggest that the predictive value of PD-L1 on tumor cells seems to be most robust in the context of advanced melanoma. ${ }^{48}$ However, responses to nivolumab have been reported in PD-L1-negative tumors. ${ }^{42}$ Moreover, data from combination studies suggest that it may be PDL1-negative patients who stand to derive proportionately more benefit from the combined ipilimumab and nivolumab treatment. $^{16}$

\section{Future directions: novel combinations and optimal sequencing}

Numerous questions around the optimal sequencing and combination of therapies for advanced melanoma remain. A body of preclinical data suggests that a potential synergy between BRAF inhibition and checkpoint blockade may exist. ${ }^{49,50}$ It has been shown that BRAF-mediated immune evasion may be reversed with BRAF inhibition without compromising T-cell function. ${ }^{51}$ Furthermore, BRAF inhibition has been reported to enhance melanoma antigen expression creating a more favorable tumor microenvironment, though an increase in tumor-infiltrating lymphocyte clonality has been reported. ${ }^{52,53}$

Currently, there is some clinical evidence suggesting that BRAF inhibition could be combined with checkpoint blockade. Retrospective analyses investigating the use of ipilimumab prior to BRAF inhibitors in $B R A F$-mutant melanoma revealed no negative influence with the use of ipilimumab prior to BRAF inhibition. ${ }^{54,55}$ Moreover, a prospective Phase II trial investigating sequential vemurafenib followed by ipilimumab demonstrated this approach to be feasible. ${ }^{56}$ However, a Phase I trial of concurrent combination of vemurafenib and ipilimumab demonstrated significant hepatotoxicity and was stopped early. ${ }^{57}$ Clinical trials examining the optimal sequence of targeted agents with immunomodulators are underway in $B R A F$-mutated melanoma. ${ }^{58} \mathrm{~A}$ recent report of an early phase clinical trial combining BRAF + MEK inhibition with the PD-L1 antibody MEDI4736 demonstrated promising response rates. ${ }^{59}$ Additionally, studies investigating nivolumab in earlier stages of melanoma, melanoma brain metastases, and rare melanoma subtypes are ongoing (Table 3).

Lastly, recent data from a Phase II study comparing nivolumab given either before or after ipilimumab in metastatic melanoma patients have shed light on the optimal sequencing of different immune checkpoint inhibitors. In this trial, a total of 140 patients were randomized to receive nivolumab induction for six cycles followed by ipilimumab for four cycles versus administration in the reverse order. The overall response rate at 25 weeks was $41 \%$ in the nivolumab induction arm versus $20 \%$ in the ipilimumab induction arm. ${ }^{60}$ Additional data are needed to help guide optimal sequencing of targeted therapy and checkpoint blockade in melanoma patients.

\section{Conclusion}

Prior to the approval of checkpoint inhibitors, metastatic melanoma patients had limited options that might provide durable responses. Encouraging results have been reported with the use of ipilimumab, nivolumab, and pembrolizumab. These and other checkpoint inhibitors are revolutionizing the management of metastatic melanoma by improving durable responses and OS.

Combination strategies will be the key in building upon these early successes. Dual immune checkpoint blockade is already commercially available, but questions around toxicity remain. $B R A F$ mutations are common in metastatic melanoma, and targeted therapy with dual BRAF/MEK inhibition now represents a standard of care option for 
Table 3 Ongoing studies using nivolumab in advanced melanoma

\begin{tabular}{|c|c|c|c|c|}
\hline Study population & Phase & Drugs & Primary outcomes & $\begin{array}{l}\text { ClinicalTrials.gov } \\
\text { identifier }\end{array}$ \\
\hline \multicolumn{5}{|l|}{ BRAF-mutated melanoma } \\
\hline Sequential checkpoint blockade & 3 & $\begin{array}{l}\text { Dab + Tram } \rightarrow \text { Nivo + Ipi vs } \\
\text { Nivo + Ipi } \rightarrow \text { Dab + Tram }\end{array}$ & Overall survival: 2 years & NCT0222478I \\
\hline CheckMate 064 & 2 & Nivo $\rightarrow$ Ipi vs Ipi $\rightarrow$ Nivo & Treatment-related Gr 3-5 AEs & NCT0I783938 \\
\hline Neoadjuvant checkpoint blockade & 2 & Nivo + lpi $\rightarrow$ Nivo vs Nivo alone & Pathologic response and pCR & NCT02519322 \\
\hline \multicolumn{5}{|l|}{ Brain metastases } \\
\hline$A B C$ & 2 & Nivo + Ipi $\rightarrow$ Nivo vs Nivo alone & Intracranial response rate & NCT02374242 \\
\hline CheckMate 204 & 2 & Nivo + lpi $\rightarrow$ Nivo & Clinical benefit rate & NCT02320058 \\
\hline \multicolumn{5}{|l|}{ Rare melanoma subtypes } \\
\hline Metastatic uveal melanoma & 2 & Nivo + Ipi $\rightarrow$ Nivo & Overall response rate & NCT0I585I94 \\
\hline KIT mutation & 2 & Sunitinib + Nivo & Objective response rate & NCT02400385 \\
\hline \multicolumn{5}{|l|}{ Correlative analyses } \\
\hline Pharmacodynamic biomarker analysis & I & Nivo + Ipi vs Nivo alone & Immunomodulatory effects & NCT0I62I 490 \\
\hline $\begin{array}{l}\text { Relationship between tumor mutation } \\
\text { burden and neoantigen load }\end{array}$ & 2 & Nivo + Ipi vs Nivo alone & Response rate & NCT02553642 \\
\hline
\end{tabular}

Abbreviations: Dab, dabrafenib; Tram, trametinib; Nivo, nivolumab; Ipi, ipilimumab; Gr, grade; AEs, adverse events; PCR, pathologic complete response; ABC, anti-PD-I brain collaboration; PD-I, programmed death-I.

these patients. Clinical trials investigating the sequencing of BRAF/MEK inhibitors with checkpoint blockade are underway and will help refine optimal sequencing.

Even though much progress has been made toward advancing the lives of our metastatic melanoma patients, a large portion of patients who receive nivolumab or pembrolizumab will ultimately have progressive disease. The development and standardization of predictive biomarkers for routine clinical use remain an unmet need. Clinically, the optimal sequence and duration of therapy are also important questions that will hopefully be answered by ongoing studies.

Nivolumab has provided encouraging results in advanced melanoma patients and has rightfully taken a center seat in the field of immunotherapy. Nivolumab continues to be incorporated into the backbone of metastatic melanoma treatment options by creating clinically relevant durable responses. Be that as it may, there are several unanswered questions requiring additional investigation using nivolumab in melanoma and other cancer subtypes that may further improve the lives of our patients.

\section{Disclosure}

April KS Salama, MD, received funding for the research from Merck, BMS, Genentech, Reata, Celldex, and Immunocore and is a consultant to BMS. The other author reports no conflicts of interest in this work.

\section{References}

1. SEER Database. Available from: http://seer.cancer.gov/statfacts $/ \mathrm{html} /$ melan.html. Accessed November 9, 2015.
2. Bajetta E, Del Vecchio M, Nova P, et al. Multicenter phase III randomized trial of polychemotherapy (CVD regimen) versus the same chemotherapy (CT) plus subcutaneous interleukin-2 and interferon-alpha2b in metastatic melanoma. Ann Oncol. 2006;17(4):571-577.

3. Eggermont AM. Advances in systemic treatment of melanoma. Ann Oncol. 2010;21(suppl 7):vii339-vii344.

4. Serrone L, Zeuli M, Sega FM, Cognetti F. Dacarbazine-based chemotherapy for metastatic melanoma: thirty-year experience overview. J Exp Clin Cancer Res. 2000;19(1):21-34.

5. Atkins MB, Lotze MT, Dutcher JP, et al. High-dose recombinant interleukin 2 therapy for patients with metastatic melanoma: analysis of 270 patients treated between 1985 and 1993. J Clin Oncol. 1999;17(7):2105-2016.

6. Shinozaki M, Fujimoto A, Morton DL, Hoon DS. Incidence of BRAF oncogene mutation and clinical relevance for primary cutaneous melanomas. Clin Cancer Res. 2004;10(5):1753-1757.

7. Chapman, $\mathrm{PB}$, Hauschild $\mathrm{A}$, Robert $\mathrm{C}$, et al. Improved survival with vemurafenib in melanoma with BRAF V600E mutation. $N$ Engl J Med. 2011;364(26):2507-2516.

8. Hauschild A, Grob JJ, Demidov LV, et al. Dabrafenib in BRAF-mutated metastatic melanoma: a multicentre, open-label, phase 3 randomised controlled trial. Lancet. 2012;380(9839):358-365.

9. Larkin J, Ascierto PA, Dréno B, et al. Combined vemurafenib and cobimetinib in BRAF-mutated melanoma. NEngl J Med.2014;371(20): $1867-1876$.

10. Robert C, Karaszewska B, Schachter J, et al. Improved overall survival in melanoma with combined dabrafenib and trametinib. N Engl J Med. 2015;372(1):30-39.

11. Long GV, Stroyakovskiy D, Gogas H, et al. Dabrafenib and trametinib versus dabrafenib and placebo for Val600 BRAF-mutant melanoma: a multicentre, double-blind, phase 3 randomised controlled trial. Lancet. 2015;386(9992):444-451.

12. Maio M, Grob JJ, Aamdal S, et al. Five-year survival rates for treatmentnaive patients with advanced melanoma who received ipilimumab plus dacarbazine in a phase III trial. J Clin Oncol. 2015;33(10):1191-1196.

13. Schadendorf D, Hodi FS, Robert C, et al. Pooled analysis of longterm survival data from phase II and phase III trials of ipilimumab in unresectable or metastatic melanoma. J Clin Oncol. 2015;33(17): 1889-1894.

14. Robert C, Schachter J, Long GV, et al. Pembrolizumab versus ipilimumab in advanced melanoma. $N$ Engl J Med. 2015;372(26): 2521-2532. 
15. Robert C, Long GV, Brady B, et al. Nivolumab in previously untreated melanoma without BRAF mutation. $N$ Engl J Med. 2015; 372(4):320-330

16. Larkin J, Chiarion-Sileni V, Gonzalez R, et al. Combined nivolumab and ipilimumab or monotherapy in untreated melanoma. N Engl J Med. 2015;373(1):23-34.

17. Andtbacka RH, Kaufman HL, Collichio F, et al. Talimogene laherparepvec improves durable response rate in patients with advanced melanoma. J Clin Oncol. 2015;33(25):2780-2788.

18. Sharma P, Allison JP. Immune checkpoint targeting in cancer therapy: toward combination strategies with curative potential. Cell. 2015;161(2):205-214.

19. Rosenberg SA. CCR 20th anniversary commentary: autologous t cells the ultimate personalized drug for the immunotherapy of human cancer. Clin Cancer Res. 2015;21(24):5409-5411.

20. Rosenberg SA, Packard BS, Aebersold PM, et al. Use of tumorinfiltrating lymphocytes and interleukin-2 in the immunotherapy of patients with metastatic melanoma. A preliminary report. $N$ Engl J Med. 1988;319(25):1676-1680.

21. Rosenberg SA, Yang JC, Sherry RM, et al. Durable complete responses in heavily pretreated patients with metastatic melanoma using $\mathrm{T}$ cell transfer immunotherapy. Clin Cancer Res. 2011;17(13):4550-4557.

22. Brahmer JR, Tykodi SS, Chow LQ, et al. Safety and activity of antiPD-L1 antibody in patients with advanced cancer. $N$ Engl J Med. 2012; 366(26):2455-2465.

23. Hamid O, Patel M, Hodi S, et al. Preliminary clinical safety, tolerability and activity of atezolizumab (anti-PD-L1) combined with Zelboraf in BRAFv600 metastatic melanoma. San Francisco, CA: Society for Melanoma Research International Congress; 2015.

24. Ishida Y, Agata Y, Shibahara K, Honjo T, et al. Induced expression of PD-1, a novel member of the immunoglobulin gene superfamily, upon programmed cell death. EMBO J. 1992;11(11):3887-3895.

25. Finger LR, Pu J, Wasserman R, et al. The human PD-1 gene: complete cDNA, genomic organization, and developmentally regulated expression in B cell progenitors. Gene. 1997;197(1-2):177-187.

26. Chemnitz JM, Parry RV, Nichols KE, June CH, Riley JL. SHP-1 and SHP-2 associate with immunoreceptor tyrosine-based switch motif of programmed death 1 upon primary human $\mathrm{T}$ cell stimulation, but only receptor ligation prevents $\mathrm{T}$ cell activation. J Immunol. 2004;173(2):945-954.

27. Dong H, Zhu G, Tamada K, Chen L. B7-H1, a third member of the B7 family, co-stimulates T-cell proliferation and interleukin-10 secretion. Nat Med. 1999;5(12):1365-1369.

28. Wang W, Carper K, Malone F, et al. PD-L1/PD-1 signal deficiency promotes allogeneic immune responses and accelerates heart allograft rejection. Transplantation. 2008;86(6):836-844.

29. Bellou A, Finn PW. Costimulation: critical pathways in the immunologic regulation of asthma. Curr Allergy Asthma Rep. 2005;5(2): $149-154$.

30. Topalian SL, Drake CG, Pardoll DM. Immune checkpoint blockade: a common denominator approach to cancer therapy. Cancer Cell. 2015;27(4):450-461.

31. Gianchecchi E, Delfino DV, Fierabracci A. Recent insights into the role of the PD-1/PD-L1 pathway in immunological tolerance and autoimmunity. Autoimmun Rev. 2013;12(11):1091-1100.

32. Iwai Y, Terawaki S, Honjo T. PD-1 blockade inhibits hematogenous spread of poorly immunogenic tumor cells by enhanced recruitment of effector T cells. Int Immunol. 2005;17(2):133-144.

33. Brahmer JR, Drake CG, Wollner I, et al. Phase I study of single-agent anti-programmed death-1 (MDX-1106) in refractory solid tumors: safety, clinical activity, pharmacodynamics, and immunologic correlates. J Clin Oncol. 2010;28(19):3167-3175.

34. Nivolumab [package insert]. http://www.accessdata.fda.gov/drugsatfda_docs/label/2014/125554lbl.pdf. Accessed November 17, 2015.

35. Topalian SL, Hodi FS, Brahmer JR, et al. Safety, activity, and immune correlates of anti-PD-1 antibody in cancer. NEngl J Med. 2012;366(26): $2443-2454$.
36. Hodi FS, O'Day SJ, McDermott DF, et al. Improved survival with ipilimumab in patients with metastatic melanoma. $N$ Engl J Med. 2010;363(8):711-723.

37. Robert C, Ribas A, Wolchok JD, et al. Anti-programmed-death-receptor-1 treatment with pembrolizumab in ipilimumab-refractory advanced melanoma: a randomised dose-comparison cohort of a phase 1 trial. Lancet. 2014;384(9948):1109-1117.

38. Topalian SL, Sznol M, McDermott DF, et al. Survival, durable tumor remission, and long-term safety in patients with advanced melanoma receiving nivolumab. J Clin Oncol. 2014;32(10):1020-1030.

39. Weber JS, D'Angelo SP, Minor D, et al. Nivolumab versus chemotherapy in patients with advanced melanoma who progressed after anti-CTLA-4 treatment (CheckMate 037): a randomised, controlled, open-label, phase 3 trial. Lancet Oncol. 2015;16(4):375-384.

40. Postow MA, Chesney J, Pavlick AC, et al. Nivolumab and ipilimumab versus ipilimumab in untreated melanoma. $N$ Engl J Med. 2015; 372(21):2006-2017.

41. Larkin J, Lao CD, Urba WJ, et al. Efficacy and safety of nivolumab in patients with BRAF V600 mutant and BRAF wild-type advanced melanoma: a pooled analysis of 4 clinical trials. JAMA Oncol. 2015;1(4): 433-440.

42. Robert C, Long GV, Brady B, et al. Nivolumab in previously untreated melanoma without BRAF mutation. NEngl JMed. 2015;372(4):320-330.

43. Wolchok JD, Kluger H, Callahan MK, et al. Nivolumab plus ipilimumab in advanced melanoma. N Engl J Med. 2013;369(2):122-133.

44. Postow M, Chesney J, Pavlick A. Initial report of overall survival rates from a randomized phase II trial evaluating the combination of nivolumab (NIVO) and ipilimumab (IPI) in patients with advanced melanoma (MEL). AACR. 2016;(suppl abstract CT002).

45. Weber JS, Kudchadkar RR, Yu B, et al. Safety, efficacy, and biomarkers of nivolumab with vaccine in ipilimumab-refractory or -naive melanoma. J Clin Oncol. 2013;31(34):4311-4318.

46. Rimm D, Schalper K, Pusztai L. Unvalidated antibodies and misleading results. Breast Cancer Res Treat. 2014;147(2):457-458.

47. Velcheti V, Schalper KA, Carvajal DE, et al. Programmed death ligand-1 expression in non-small cell lung cancer. Lab Invest. 2014;94(1): 107-116.

48. Carbognin L, Pilotto S, Milella M, et al. Differential activity of nivolumab, pembrolizumab and MPDL3280A according to the tumor expression of programmed death-ligand-1 (PD-L1): sensitivity analysis of trials in melanoma, lung and genitourinary cancers. PLoS One. 2015;10(6):e0130142.

49. Kim T, Amaria RN, Spencer C, et al. Combining targeted therapy and immune checkpoint inhibitors in the treatment of metastatic melanoma. Cancer Biol Med. 2014;11(4):237-246.

50. Wargo JA, Cooper ZA, Flaherty KT. Universes collide: combining immunotherapy with targeted therapy for cancer. Cancer Discov. 2014; 4(12):1377-1386.

51. Boni A, Cogdill AP, Dang P, et al. Selective BRAFV600E inhibition enhances T-cell recognition of melanoma without affecting lymphocyte function. Cancer Res. 2010;70(13):5213-5219.

52. Cooper ZA, Frederick DT, Juneja VR, et al. BRAF inhibition is associated with increased clonality in tumor-infiltrating lymphocytes. Oncoimmunology. 2013;2(10): 26615.

53. Frederick DT, Piris A, Cogdill AP, et al. BRAF inhibition is associated with enhanced melanoma antigen expression and a more favorable tumor microenvironment in patients with metastatic melanoma. Clin Cancer Res. 2013;19(5):1225-1231.

54. Ackerman A, Klein O, McDermott DF, et al. Outcomes of patients with metastatic melanoma treated with immunotherapy prior to or after BRAF inhibitors. Cancer. 2014;120(11):1695-1701.

55. Ascierto PA, Margolin K. Ipilimumab before BRAF inhibitor treatment may be more beneficial than vice versa for the majority of patients with advanced melanoma. Cancer. 2014;120(11):1617-1619.

56. Amin A, Lawson DH, Salama AK, et al. A single-arm, open-label, phase II study to evaluate the safety of vemurafenib (VEM) followed by ipilimumab (IPI) in BRAF V600-mutated metastatic melanoma (MM). J Clin Oncol. 2015;33 (suppl; abstr 9032). 
57. Ribas A, Hodi FS, Callahan M, Konto C, Wolchok J. Hepatotoxicity with combination of vemurafenib and ipilimumab. N Engl J Med. 2013; 368(14):1365-1366.

58. Atkins M; National Cancer Institute. Dabrafenib and trametinib followed by ipilimumab and nivolumab or ipilimumab and nivolumab followed by dabrafenib and trametinib in treating patients with stage III-IV BRAFV600 melanoma. https://clinicaltrials.gov/ct2/show/study/ NCT02224781. Accessed July 19, 2016.
59. Ribas A. Phase I study combining anti-PD-L1 (MEDI4736) with BRAF (dabrafenib) and/or MEK (trametinib) inhibitors in advanced melanoma. J Clin Oncol. 2015;33(suppl; abstr 3003).

60. Hodi FS, Gibney G, Sullivan R, et al. An open-label, randomized, phase 2 study of nivolumab (NIVO) given sequentially with ipilimumab (IPI) in patients with advanced melanoma (CheckMate 064). Presented at: European Cancer Congress; 2015; Vienna, Austria.

\section{Publish your work in this journal}

ImmunoTargets and Therapy is an international, peer-reviewed open access journal focusing on the immunological basis of diseases, potential targets for immune based therapy and treatment protocols employed to improve patient management. Basic immunology and physiology of the immune system in health, and disease will be also covered. In addition, the journal will focus on the impact of manage- ment programs and new therapeutic agents and protocols on patient perspectives such as quality of life, adherence and satisfaction. The manuscript management system is completely online and includes a very quick and fair peer-review system, which is all easy to use. Visit http://www.dovepress.com/testimonials.php to read real quotes from published authors.

Submit your manuscript here: http://www.dovepress.com/immunotargets-and-therapy-journal 